\title{
Quadrature Rule Methods for Volterra Integral Equations of the First Kind
}

\author{
By Charles J. Gladwin
}

\begin{abstract}
A new class of quadrature rule methods for solving nonsingular Volterra integral equations of the first kind are introduced; these methods are based on an appropriate modification of the higher-order Newton-Gregory methods which are known to be divergent. Methods up to order six are constructed explicitly and illustrated with numerical examples.
\end{abstract}

1. Introduction. Consider the linear Volterra integral equation of the first kind,

$$
\int_{0}^{x} K(x, t) y(t) d t=f(x),
$$

where $f$ and $K$ (the kernel) are given functions which are continuously differentiable to sufficiently high order with respect to each of their arguments on

$$
S_{1}=\{x \mid 0 \leqslant x \leqslant a\} \quad(a<\infty),
$$

and on

$$
S_{2}=\{(x, t) \mid 0 \leqslant t \leqslant x \leqslant a\},
$$

respectively. Furthermore, we assume that

(1) $f(0)=0$, and

(2) $K(x, x) \neq 0$ for all $x \in S_{1}$.

Then it is well known, [11, p. 14], that a unique solution $y(x)$ exists and is continuously differentiable to sufficiently high order on $S_{1}$.

2. Method of Solution. We introduce the uniform mesh

$$
I_{N}=\left\{x_{n}=n h \mid n=0(1) N, N h=a\right\}
$$

on the basic interval $S_{1}$. The integral in (1.1) is then replaced by a quadrature rule of the form

$$
\int_{0}^{x_{n}} g(x) d x=h \sum_{i=0}^{n} w_{n i} g\left(x_{i}\right)+E_{n}(g)
$$

where $w_{n i}$ are real-valued weights satisfying

Received March 17, 1975; revised December 29, 1975, August 23, 1976, April 14, 1977 and August 8, 1978.

AMS (MOS) subject classifications (1970). Primary 65R05; Secondary 45L10.

Key words and phrases. Quadrature rule methods, Volterra integral equations of the first kind.

(C) 1979 American Mathematical Society 0025-571 8/79/0000-0065/\$04.00 


$$
w_{n i}= \begin{cases}a_{i}^{*}, & i=0(1) p \\ 1, & i=p+1(1) n-(p+1) \\ a_{n-i}, & i=n-p(1) n .\end{cases}
$$

Equation (2.1) will be denoted by $Q\left(A^{*}, A, m\right)$, where

(1) $A^{*}=\left(a_{i}^{*}\right) \in R^{p+1}, A=\left(a_{i}\right) \in R^{p+1}$.

(2) $m$ is the degree of precision of the quadrature rule [8, p. 301],

i.e. $E_{n}(g)=0 \forall g \in \pi_{m}$, space of real polynomials of degree $m$.

(3) (2.1) is "apparently" defined for $n \geqslant 2 p+1$. In Section 3, we shall extend the definition for $n \geqslant p+1$.

This yields the numerical method for the solution of (1.1),

$$
\sum_{i=0}^{n} w_{n i} K\left(x_{n}, x_{i}\right) y_{i}=f\left(x_{n}\right), \quad n=p+1(1) N
$$

where $y_{n}$ denotes an approximation to the exact solution $y\left(x_{n}\right)$. If we have a set of starting values $y_{n}, n=0(1) p$, then (2.2) may be used in a recursive fashion to generate approximations for $y_{n}, n=p+1(1) N$.

In this paper we shall not deal with the problem of how to generate the necessary starting values; we mention, however, that some useful higher-order procedures have recently been derived in [1, p. 295] and [13, p. 45]. These methods could also be used to approximate $y$ on the entire mesh $I_{N}$; however, they are $r^{2}$ times as expensive (in terms of the number of kernel evaluations per step) as the method defined in (2.2) ( $r$ is the order of the method, to be defined below).

Definition 2.1. Let $e_{n}=y_{n}-y\left(x_{n}\right), n=0(1) N$, denote the (global) discretization error of (2.2). Then (2.2) is said to be convergent of order $r$ if $r$ is the largest positive integer for which there exists a finite constant $C$ such that, for all sufficiently smooth $f$ and $K$,

$$
\left|e_{n}\right| \leqslant C h^{r}, \quad n=0(1) N .
$$

The aim of this paper will be to give a characterization of the weights $w_{n i}$ so that the method (2.2) is actually convergent of order $r(=m+1$, as we shall see later). Furthermore, we shall construct explicitly families of such methods for $r \leqslant 6$.

This is significant in the light of the result in [5, p. 148], which essentially showed that methods of the form (2.2) based on a wide class of interpolatory quadrature rules yielded nonconvergent methods for $r>2$.

It should be mentioned, however, that six interpolatory quadrature rules were displayed in $[5$, p. 148] which proved to yield convergent schemes up to order six. Unfortunately, these schemes give no indication as to whether methods of higher order exist.

3. Selection of the Weights. In order to choose the vectors $A^{*}$ and $A$ we consider the decomposition of (2.1) into simpler quadrature rules 


$$
\int_{0}^{x_{n}} g(x) d x=\int_{0}^{x_{p}} g(x) d x+\sum_{j=p+1}^{n} \int_{x_{j-1}}^{x_{j}} g(x) d x
$$

$$
=\left\{h \sum_{i=0}^{p} c_{i} g\left(x_{i}\right)+\hat{E}_{p}(g)\right\}+\sum_{j=p+1}^{n}\left\{h \sum_{i=0}^{p+1} b_{i} g\left(x_{j-i}\right)+\hat{E}_{j}(g)\right\},
$$

where

$$
E_{n}(g)=\hat{E}_{p}(g)+\sum_{j=p+1}^{n} \hat{E}_{j}(g)
$$

Now we select:

(1) The $c_{i}$ to be the weights of the closed Newton-Cotes rule with $p+1$ abscissae.

(2) ${ }^{\circ}$ The $b_{i}$ to satisfy the system of linear equations

$$
\sum_{i=0}^{p+1} i^{j} b_{i}=\frac{1}{j+1}, \quad j=0(1) r-1 \leqslant p+1 \quad\left(0^{j}=\left\{\begin{array}{ll}
1, & j=0 \\
0, & j>0
\end{array}\right) .\right.
$$

(In the following, conditions (3.2) will be referred to as the consistency conditions.)

With these choices, it follows that the degree of precision of (2.1) is $m=r-1$.

Furthermore, if $r-1=p+1$, the system (3.2) yields a unique solution (since the coefficient matrix is essentially Vandermonde). In this case, the quadrature rule (2.1) is the Newton-Gregory rule to $p$ th differences. This case is useless for $p>0$; see $[5$, p. 148] or $[6$, p. 38]. Thus, in the remainder of the paper we shall assume $r-1$ $<p+1$ so that the weights of (2.1) will depend (linearly) on $p+2-r$ free parameters $b_{0}, b_{1}, b_{2}, \ldots, b_{p+1-r}$, say.

Finally, we associate with (2.1) the characteristic polynomial

$$
\rho(z)=\sum_{i=0}^{p+1} b_{i} z^{p+1-i}
$$

4. Convergence of the Method. We need the following definition.

Definition $4.1\left[10\right.$, p. 398]. Let $\rho(z) \in \pi_{n}$. Then

(1) $\rho(z)$ is said to be a simple von Neumann polynomial if

(a) $\rho(z)=0 \Rightarrow|z| \leqslant 1$,

(b) $\rho(z)=0$ and $|z|=1 \Rightarrow \rho^{\prime}(z) \neq 0$.

(2) $\rho(z)$ is a Schur polynomial if $\rho(z)=0 \Rightarrow|z|<1$.

We now give necessary conditions for (2.2) to define a convergent method of order 1 .

THEOREM 4.1. Assume (2.2) is convergent of order 1. Then the polynomial $\rho(z)$, defined in (3.3), satisfies:

(1) $\rho(z)$ is a simple von Neumann polynomial (stability condition), and

(2) $\rho(1)=1(j=0$ in (3.2)) (consistency condition).

Proof. For (1), we apply (2.2) to the test equation

$$
\int_{0}^{x} y(t) d t=0, \quad y(x) \equiv 0
$$


to obtain

$$
h \sum_{i=0}^{p} c_{i} y_{i}+\sum_{j=p+1}^{n} h \sum_{i=0}^{p+1} b_{i} y_{j-i}=0 .
$$

Backward differencing of this relation at $n$ and $n-1$ yields the difference equation

$$
\sum_{i=0}^{p+1} b_{i} y_{n-i}=0, \quad n=p+1(1) N .
$$

If the method is convergent, we must have

$$
\lim _{h \rightarrow 0} y_{n}=0=y\left(x_{n}\right) \quad\left(n \rightarrow \infty, n h=x_{n} \in S_{1}\right),
$$

which entails that $\rho(z)$ must be a simple von Neumann polynomial. See [7, p. 218] for the details.

For (2), we apply the same procedure to the test equation

$$
\int_{0}^{x} y(t) d t=x
$$

(whose exact solution is $y(x) \equiv 1$ ) to obtain the difference equation

$$
\sum_{i=0}^{p+1} b_{i} y_{n-i}=1, \quad n=p+1(1) N .
$$

Thus, the result follows.

We now proceed to show that stability and consistency are sufficient for convergence. Indeed, we shall show if we have the $r$ conditions (3.2), we obtain convergence of order $r$.

Throughout the rest of the paper we assume that the following conditions hold:

A. $f$ and $K$ are sufficiently differentiable with respect to each of their arguments on $S_{1}$ and $S_{2}$, respectively.

B. $\rho(z)$, defined in (3.3), satisfies the stability condition and the $r$ consistency conditions (3.2) (i.e. (3.2) with $j=0,1, \ldots, r-1$ ).

C. The starting errors, $\left\{e_{n}: n=O(1) p\right\}$, are $O\left(h^{r}\right)$.

It is easily seen that the global discretization error, $e_{n}$, satisfies

$$
\begin{array}{r}
h \sum_{i=0}^{p} c_{i} K\left(x_{n}, x_{i}\right) e_{i}+h \sum_{j=p+1}^{n} \sum_{i=0}^{p+1} b_{i} K\left(x_{n}, x_{j-i}\right) e_{j-i}=E_{n} \\
(n=p+1(1) N),
\end{array}
$$

where $E_{n}$ is the quadrature rule error in (2.1) applied to the function $K\left(x_{n}, t\right) \cdot y(t)$. $E_{n}$ can be estimated asymptotically $\left(n \rightarrow \infty, h \rightarrow 0, n h=x_{n}\right.$ fixed), under assumptions $\mathrm{A}, \mathrm{B}$ and $\mathrm{C}$, by

$$
E_{n}=h^{r} \phi_{1}\left(x_{n}\right)+h^{r+1} \phi_{2}\left(x_{n}\right)+h^{r+2} \phi_{3}\left(x_{n}\right)+O\left(h^{r+3}\right)
$$

with (a) $\phi_{i} \in C^{2}\left[S_{1}\right], i=1(1) 3$, (b) $\phi_{1}(0)=0$.

We shall also need the following lemma.

Lemma 4.1. Let $P(z)$ be a real simple von Neumann polynomial (with $P(1) \neq 0$ ) of exact degree $q$ and with no zeros at the origin. Furthermore, let the coefficients 
$\left\{\gamma_{l}: l=0(1)^{\infty}\right\}$ be defined by

$$
\frac{1}{{ }_{z^{q} P(1 / z)}}=\sum_{l=0}^{\infty} \gamma_{l} z^{l}
$$

Then, for any $\phi \in C^{1}\left[S_{1}\right]$, we have

$$
\left|\sum_{l=0}^{n} \gamma_{l} \phi\left(x_{l}\right)\right| \leqslant M<\infty \text { for all } n=0(1) N ; x_{l}=l h
$$

Proof. Let $P(z)=\Sigma_{i=0}^{q} b_{i} z^{q-i}$. Then from (4.3) we obtain

$$
\sum_{i=0}^{q} b_{i} \gamma_{l-i}= \begin{cases}1, & l=0 \\ 0, & l>0\end{cases}
$$

with $\gamma_{l-i}=0$ for $l<i$. For $l \geqslant q,(4.4)$ is a linear, homogeneous difference equation with constant coefficients together with $q$ starting values $\left\{\gamma_{l}: l=O(1) q-1\right\}$. Thus, using the hypotheses on $P(z)$, we may write

$$
\gamma_{l}=\sum_{i=1}^{s} C_{i} z_{i}^{l}+D_{1} \mu^{l}+\sum_{i=2}^{V} D_{i} l(l-1) \cdots(l-i+2) \mu^{l}, \quad l \geqslant 0
$$

where (a) $C_{i}, D_{i}$ are arbitrary constants, depending linearly on the starting values; (b) $P(z)$ is assumed (for ease of notation and without loss of generality) to have $s$ simple zeros, $z_{i}$, on the unit circle and one zero, $\mu$, of multiplicity $v$ interior to the unit circle; here, $s+v=q$.

Thus, it suffices to see that

$$
\left|\sum_{l=0}^{n} z^{l} \phi(l h)\right| \leqslant M^{*}<\infty \quad \text { for all } n=0(1) N,|z|=1, z \neq 1
$$

We have

where

$$
\sum_{l=0}^{n} z_{l} \phi\left(x_{l}\right)=\sum_{l=0}^{n}\left(S_{l}-S_{l-1}\right) \phi\left(x_{l}\right), \quad x_{l}=l h
$$

$$
S_{n}=\sum_{j=0}^{n} z^{j}=\frac{z^{n+1}-1}{z-1}, \quad S_{-1}=0, \quad S_{0}=0,
$$

or, using summation by parts,

$$
\sum_{l=0}^{n} z^{l} \phi\left(x_{l}\right)=\sum_{l=0}^{n-1} S_{l}\left[\phi\left(x_{l}\right)-\phi\left(x_{l+1}\right)\right]+S_{n} \cdot \phi\left(x_{n}\right) .
$$

The result now follows by applying the Mean Value Theorem of Differential Calculus. See [7, p. 269] for the details.

We are now prepared to show that stability and consistency are sufficient for convergence.

THEOREM 4.2. Assume that $e_{n}$ satisfies (4.1) and that the conditions A, B and $\mathrm{C}$ are fulfilled. Then the method defined by (2.2) is convergent of order $r$; i.e.

$$
e_{n}=O\left(h^{r}\right), \quad n=O(1) N, \text { as } h \rightarrow 0, N h=a \text {. }
$$


Proof. By using backward differencing in (4.1), and by observing Taylor's formula and assumption $\mathbf{A}$, together with

(a) $K_{n \cdot i}=K\left(x_{n}, x_{i}\right), i=0(1) n$,

(b) $K_{n \cdot n-i}=K_{n \cdot n}-i h \theta_{n \cdot i},\left|\theta_{n \cdot i}\right| \leqslant \theta=\max _{S_{2}}\left\{\left|K_{t}\right|\right\}$,

(c) $K_{n-1 \cdot i}=K_{n \cdot i}-h \chi_{n \cdot i}+\theta_{n \cdot i}^{*} h^{2}$,

$$
\left|\chi_{n \cdot i}\right| \leqslant \chi=\max _{S_{2}}\left\{\left|K_{x}\right|\right\}, \quad\left|\theta_{n \cdot i}^{*}\right| \leqslant \theta^{*}=\max _{S_{2}}\left\{\left|K_{x x}\right|\right\},
$$

(d) $\left|K_{n n}\right| \geqslant \lambda>0$ for all $n=0(1) N$,

we obtain

$$
\sum_{i=0}^{p+1} b_{i} e_{n-i}=K_{n \cdot n}^{-1}\left\{h \sum_{i=0}^{n-1} d_{n \cdot i} e_{i}+h^{-1}\left(E_{n}-E_{n-1}\right)\right\}, \quad n=p+1(1) N,
$$

where

$$
d_{n \cdot i}= \begin{cases}\left(-\chi_{n \cdot i}+\theta_{n \cdot i}^{*} h\right) s_{i}, & i=0(1) p, \\ \left(-\chi_{n \cdot i}+\theta_{n \cdot i}^{*} h\right), & i=p+1(1) n-(p+2), \\ \left(-\chi_{n \cdot i}+\theta_{n \cdot i}^{*}\right) t_{i}+u_{i} \theta_{n n-i}, & i=n-(p+1)(1) n-1,\end{cases}
$$

and where $s_{i}, t_{i}, u_{i}$ depend (linearly) only on $c_{i}$ and $b_{i}$.

Here, the functions $d_{n \cdot i}=d_{i}\left(x_{n}\right)$ are sufficiently smooth in view of assumption A. Multiply (4.6) by $\gamma_{N-n}$ for each $n=p+1(1) N$ and form the sum. We first note that (from (4.4))

$$
\sum_{n=p+1}^{N} \gamma_{N-n} \sum_{i=0}^{p+1} b_{i} e_{n-i}=e_{N}+\sum_{k=1}^{p+1} \sum_{i=k}^{p+1} b_{k} \gamma_{N-(p+k)} e_{p+k-i}
$$

Secondly, we have

$$
\begin{aligned}
& \sum_{n=p+1}^{N} \gamma_{N-n} K_{n n}^{-1} \sum_{i=0}^{n-1} d_{n \cdot i} e_{i} \\
& \quad=\sum_{i=0}^{p}\left\{\sum_{n=p+1}^{N} \gamma_{N-n} K_{n n}^{-1} d_{n \cdot i}\right\} e_{i}+\sum_{i=p+1}^{N-1} \gamma_{N \cdot i}^{*} e_{i},
\end{aligned}
$$

where $\gamma_{N \cdot i}^{*}=\Sigma_{n=i+1}^{N} \gamma_{N-n} K_{n n}^{-1} d_{n \cdot i}, i=p+1(1) N-1$. We note

(a) $\left|\Sigma_{n=p+1}^{N} \gamma_{N-n} K_{n n}^{-1} d_{n \cdot i}\right| \leqslant \Gamma_{1}<\infty$, and

(b) $\left|\gamma_{N \cdot i}^{*}\right| \leqslant \Gamma_{2}<\infty$,

for all $i$ and $N$, in view of Lemma 4.1 .

Collecting the above results, we find

$$
\left|e_{N}\right| \leqslant h S \sum_{i=0}^{N-1}\left|e_{i}\right|+R, \quad N \geqslant 0,
$$

where $S=\max \left\{\Gamma_{1}, \Gamma_{2}\right\}$,

$$
R=\sum_{k=1}^{p+1} \sum_{i=k}^{p+1}\left|b_{k}\right|\left|\gamma_{N-(p+k)} \| e_{p+k-i}\right|+\left|\sum_{n=p+1}^{N} \gamma_{N-n} K_{n n}^{-1} h^{-1}\left(E_{n}-E_{n-1}\right)\right| \text {. }
$$

The double sum is finite and $O\left(h^{r}\right)$, since it only depends on the starting errors which, due to assumption C, satisfy $e_{n}=O\left(h^{r}\right)(n=O(1) p)$. The second sum is $O\left(h^{r}\right)$, 
too, which may be easily verified by inserting (4.2) (using appropriate backward differencing), applying the Mean Value Theorem, and observing Lemma 4.1.

Thus the solution of the recursive inequality $(4.8)$ is $[7$, p. 244],

$$
\left|e_{N}\right| \leqslant R(1+S h)^{N} \leqslant R \cdot \exp (N h S), \quad N h=a,
$$

so that $\left|e_{N}\right|=O\left(h^{r}\right)$ for $n=O(1) N$. Thus the proof is complete.

5. Construction of Methods. We first consider the choice of the $p+2-r$ free parameters so that $\rho(z)$ is a Schur polynomial. An equivalent (and more convenient) approach is to ensure that the polynomial

$$
\tau(w)=(w-1)^{p+1} \rho\left(\frac{w+1}{w-1}\right)=\sum_{j=0}^{p+1} \tau_{j} w^{j}
$$

is a Hurwitz polynomial [3, p. 206]. (A Hurwitz polynomial is a polynomial such that $\tau(w)=0 \Rightarrow \operatorname{Re} w<0$.)

It is easy to see $[3$, p. 204] that

$$
\tau_{j}=\sum_{i=0}^{p+1} b_{p+1-i} \gamma_{i j}, \quad j=0(1) p+1,
$$

where $\gamma_{i j}=\gamma_{i \cdot j-1}-\gamma_{i-1 \cdot j-1}-\gamma_{i-1 \cdot j}(i, j=1(1) p+1)$, with

$$
\begin{gathered}
\gamma_{i \cdot 0}=(-1)^{p+1-i}, \quad \gamma_{0 \cdot j}=\left(\begin{array}{c}
p+1 \\
j
\end{array}\right)(-1)^{p+1-j}, \\
\gamma_{i \cdot p+1}=1, \quad \gamma_{p+1 \cdot j}=\left(\begin{array}{c}
p+1 \\
j
\end{array}\right),
\end{gathered}
$$

where we have used (3.3).

Remark. It can be easily shown that, for $\rho(z)$ satisfying (3.2) and (3.3)

(i) $\partial \tau_{i} / \partial b_{0}=0, i=1(1) p+1, r=p+1$,

(ii) $\partial \tau_{i} / \partial b_{0}=\partial \tau_{i} / \partial b_{1}=0, i=2(1) p+1, r=p$.

This permits the use of Theorem 5.1 which is a simpler form of the usual Hurwitz criterion. It should be noted, however, that Theorem 5.1 is valid for arbitrary real polynomials. In order to introduce a Hurwitz criterion, we define the sequence of determinants

$$
\delta_{k}=\left|\begin{array}{ccccc}
\tau_{1} & \tau_{3} & \cdots & \tau_{2 k-1} \\
\tau_{0} & \tau_{2} & \cdots & \tau_{2 k-2} \\
0 & \tau_{1} & \cdots & \tau_{2 k-3} \\
0 & \tau_{0} & \cdots & \tau_{2 k-4} \\
\cdot & \cdot & & \cdot \\
\cdot & \cdot & & \cdot \\
\cdot & \cdot & & \cdot \\
0 & 0 & \cdots & \tau_{k}
\end{array}\right|, \quad k=1(1) p+1\left(\tau_{j}=0 \text { if } j>p+1\right)
$$

where $\tau_{j}$ are as in (5.1). 
The reader should also note that $\tau(w)$ is a Hurwitz polynomial if and only if

$$
\tau^{*}(w)=w^{p+1} \tau\left(\frac{1}{w}\right)=\sum_{j=0}^{p+1} \tau_{j} w^{p+1-j}
$$

is a Hurwitz polynomial. We then have the following result.

THEOREM 5.1. The following conditions are necessary and sufficient for $\tau^{*}(w)$ to be a Hurwitz polynomial:

(i) $\tau_{p+1}>0, \tau_{p-1}>0, \tau_{p-3}>0, \ldots ; \delta_{p}>0, \delta_{p-2}>0, \ldots$, or

(ii) $\tau_{p+1}>0, \tau_{p}>0, \tau_{p-2}>0, \ldots ; \delta_{p}>0, \delta_{p-2}>0, \ldots$

Proof $[2$, p. 158]. To construct a simple von Neumann polynomial we chose the free parameters so that

(a) $\tau(0)=0$,

(b) $\tau(w) / w$ is a Hurwitz polynomial.

\section{TABLE I}

\begin{tabular}{|c|c|c|c|}
\hline \multirow{7}{*}{$\begin{array}{l}\rho(z): \\
\text { SCHUR }\end{array}$} & $r$ & $\mathrm{p}$ & DOMAIN OF STABILITY \\
\hline & 1 & 0 & $b_{0}>1 / 2$ \\
\hline & 2 & 1 & $b_{0}>1 / 2$ \\
\hline & 3 & 2 & $11 / 24<b_{0}<15 / 24$ \\
\hline & 4 & 4 & $b_{0}=0,45 / 24<b_{1}<77 / 24$ \\
\hline & 5 & 5 & $\begin{array}{l}b_{0}=0 \\
3251 / 1440<b_{1}<\left(-14518 / 5+7(289845)^{\frac{1}{2}}\right) / 288\end{array}$ \\
\hline & 6 & 6 & $\begin{array}{l}b_{0}=0 \\
941 / 360<b_{1}<133 / 60+(1005)^{\frac{1}{2}} / 40\end{array}$ \\
\hline \multirow{7}{*}{$\begin{array}{l}\rho(z): \\
\text { SIMPLE } \\
\text { VON NEUMANN }\end{array}$} & $r$ & p & DOMAIN OF STABILITY \\
\hline & 1 & 1 & $b_{0}>1 / 4, b_{1}=1 / 2$ \\
\hline & 2 & 2 & $b_{0}>3 / 8, b_{0}+b_{1}=1$ \\
\hline & 3 & 3 & $5 / 12<b_{0}<11 / 12,16 b_{0}+8 b_{1}=35 / 3$ \\
\hline & 4 & 4 & $5 / 12<b_{0}<25 / 48,3 b_{0}+b_{1}=15 / 8$ \\
\hline & 5 & 5 & $\begin{aligned} 1151 / 2880<b_{0}<-1199 / 480+(5 / 8)(1307 / 60)^{\frac{1}{2}} \\
128 b_{0}+32 b_{1}=3251 / 45\end{aligned}$ \\
\hline & 6 & 6 & $\begin{array}{l}b_{0}=0 \\
b_{1}=941 / 360\end{array}$ \\
\hline
\end{tabular}

For $p=3$ and $r=4 ; \tau_{1}=0$ in the polynomial $\tau(w)$. Thus, $\rho(z)$ cannot be a Schur polynomial for any $b_{0}[3$, p. 198]. 
Conditions (a) and (b) are obviously sufficient for $\rho(z)$ to have a single root at $z=-1$ and $p$ roots in the interior of $|z|=1$.

In the following table we list a number of polynomials $\rho$ satisfying the conditions mentioned above; the derivation of these results is then illustrated by means of two examples.

Examples. 1. $\rho(z)$ is a Schur polynomial, with $r=3, p=2$. Here,

$$
\rho(z)=b_{0} z^{3}+\left(\frac{23}{12}-3 b_{0}\right) z^{2}+\left(3 b_{0}-\frac{16}{12}\right) z+\left(\frac{5}{12}-b_{0}\right)
$$

and

$$
\tau(w)=\left(8 b_{0}-\frac{44}{12}\right)+\frac{2}{3} w+2 w^{2}+w^{3} .
$$

For Theorem 5.1(ii) we use

$$
\tau_{3}=1, \quad \tau_{2}=2, \quad \tau_{1}=\frac{2}{3}, \quad \tau_{0}=8 b_{0}-\frac{44}{12},
$$

and obtain

$$
\delta_{2}=\left|\begin{array}{cc}
\frac{2}{3} & 1 \\
8 b_{0}-\frac{44}{12} & 2
\end{array}\right|=\frac{60}{12}-8 b_{0}
$$

which then yields the condition stated in Table I.

2. $\rho(z)$ is a simple von Neumann polynomial, with $r=2, p=2$. Here,

and

$$
\rho(z)=b_{0} z^{3}+b_{1} z^{2}+\left(\frac{5}{2}-3 b_{0}-2 b_{1}\right) z+\left(2 b_{0}+b_{1}-\frac{3}{2}\right),
$$

$$
\tau(w)=\left(4-4 b_{0}-4 b_{1}\right)+\left(12 b_{0}+4 b_{1}-7\right) w+2 w^{2}+w^{3}
$$

Thus, condition (a) yields

$$
4-4 b_{0}-4 b_{1}=0
$$

while (b) implies

$$
12 b_{0}+4 b_{1}-7>0
$$

which may be written as

$$
12 b_{0}+4\left(1-b_{0}\right)-7>0 .
$$

6. Examples and Conclusions. It can be shown that the global discretization error, $e_{n}$, contains terms of the form $z_{k}^{n} d_{k}\left(x_{n}\right)$, where $z_{k}$ are the roots of $\rho(z)$ and $d_{k}(x)$ are solutions of the initial value problems

$$
K(x, x) d_{k}^{\prime}(x)-z_{k} K_{t}(x, x) d_{k}(x)=0, \quad d_{k}\left(x_{0}\right)=\delta_{k}
$$

(See [6] for details; compare also Kobayasi [9] where a corresponding analysis is given for the trapezoidal method.) Thus, in the case of quadrature rule methods based on simple von Neumann polynomials, we get "marginally stable" approximations depending on whether $\left|d_{k}(x)\right|$ is monotone increasing or decreasing (with increasing $x$ ). 
TABLE II(a)

$$
\left\{\begin{array}{c}
\left|e_{n}\right| \\
\left|e_{n+1}\right|
\end{array}\right\} \text { at } x_{n}=2
$$

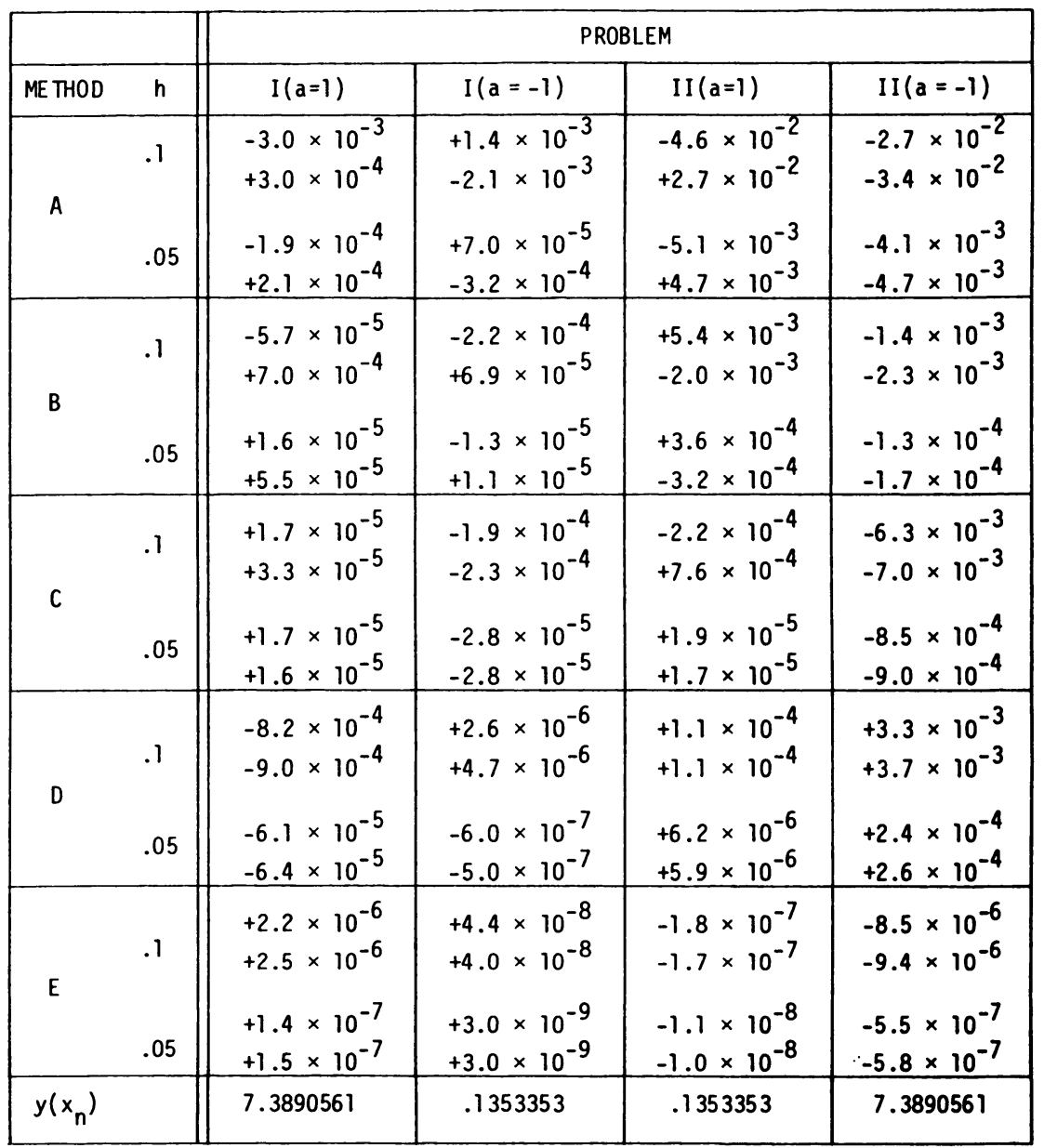

We shall illustrate this phenomenon with the following examples.

I. $\int_{0}^{x}\left(a^{2}+1\right) \cos (x-t) y(t) d t=a e^{a x}+\sin x-a \cos x, y(x)=e^{a x}, d_{k}(x)=$ $\delta_{k}$.

II. $a \int_{0}^{x} e^{a(x-t)} y(t) d t=\sinh a x, y(x)=e^{-a x}, d_{k}(x)=\delta_{k} e^{a x}, z_{k}=-1$ (for our methods).

Thus, we expect serious stability problems in example II with $a>0$.

The following methods will be used for the solution.

A: $\quad \rho(z)$ (simple von Neumann polynomial): $r=p=3, b_{0}=\frac{3}{4}, b_{1}=\frac{-1}{24}$;

B: $\rho(z)$ (simple von Neumann polynomial): $r=p=4, b_{0}=\frac{1}{2}, b_{1}=\frac{3}{8}$;

C: $\rho(z)$ (Schur polynomial): $r=3, \quad p=2, \quad b_{0}=\frac{1}{2}$; 
D: $\rho(z)$ (Schur polynomial): $r=p=4, \quad b_{0}=0, \quad b_{1}=\frac{54}{24}$;

E: block method: $u_{i}=\frac{i}{4}, i=1(1) 4$ (see Weiss [13, p. 51]).

Exact starting values were used, when needed. All computations were performed on the CDC 6400 at Dalhousie University (in double precision 120 bit word).

$$
\begin{gathered}
\text { TABLE II(b) } \\
\left\{\begin{array}{c}
\left|e_{n}\right| \\
\left|e_{n+1}\right|
\end{array}\right\} \text { at } x_{n}=4
\end{gathered}
$$

\begin{tabular}{|c|c|c|c|c|c|}
\hline & & \multicolumn{4}{|c|}{ PROBLEM } \\
\hline METHOD & $h$ & $I(a=1)$ & $I(a=-1)$ & II $(a=1)$ & $\operatorname{II}(a=-1)$ \\
\hline A & .05 & $\begin{array}{l}-1.7 \times 10^{-2} \\
-1.6 \times 10^{-2} \\
-1.1 \times 10^{-3} \\
-8.6 \times 10^{-4}\end{array}$ & $\begin{array}{r}+2.8 \times 10^{-4} \\
-1.9 \times 10^{-3} \\
+2.8 \times 10^{-5} \\
-3.1 \times 10^{-4}\end{array}$ & $\begin{array}{l}-2.3 \times 10^{0} \\
+2.1 \times 10^{0} \\
-3.5 \times 10^{-2} \\
+3.6 \times 10^{-2} \\
\end{array}$ & $\begin{array}{l}-2.2 \times 10^{-1} \\
-2.5 \times 10^{-1} \\
-3.2 \times 10^{-2} \\
-3.3 \times 10^{-2}\end{array}$ \\
\hline B & .05 & $\begin{array}{r}+3.2 \times 10^{-3} \\
+4.1 \times 10^{-3} \\
+2.5 \times 10^{-4} \\
+3.0 \times 10^{-4} \\
\end{array}$ & $\begin{array}{l}-1.3 \times 10^{-4} \\
+7.2 \times 10^{-5} \\
-1.2 \times 10^{-5} \\
+1.1 \times 10^{-5} \\
\end{array}$ & $\begin{array}{l}+2.8 \times 10^{-2} \\
-2.6 \times 10^{-2} \\
+2.7 \times 10^{-3} \\
-2.9 \times 10^{-3} \\
\end{array}$ & $\begin{array}{r}-1.4 \times 10^{-2} \\
-1.6 \times 10^{-2} \\
-1.1 \times 10^{-3} \\
-1.1 \times 10^{-3}\end{array}$ \\
\hline C & .05 & $\begin{array}{l}-1.6 \times 10^{-3} \\
-1.8 \times 10^{-3} \\
-9.3 \times 10^{-5} \\
-1.0 \times 10^{-4}\end{array}$ & $\begin{array}{l}-2.0 \times 10^{-4} \\
-2.0 \times 10^{-4} \\
-2.8 \times 10^{-5} \\
-2.8 \times 10^{-5}\end{array}$ & $\begin{array}{l}+6.1 \times 10^{-5} \\
-2.5 \times 10^{-5} \\
+2.5 \times 10^{-6} \\
+2.4 \times 10^{-6}\end{array}$ & $\begin{array}{l}-4.7 \times 10^{-2} \\
-5.2 \times 10^{-2} \\
-6.3 \times 10^{-3} \\
-6.6 \times 10^{-3}\end{array}$ \\
\hline D & .05 & $\begin{array}{l}-6.0 \times 10^{-3} \\
-6.6 \times 10^{-3} \\
-4.5 \times 10^{-4} \\
-4.7 \times 10^{-5}\end{array}$ & $\begin{array}{l}+2.7 \times 10^{-5} \\
+2.7 \times 10^{-5} \\
-7.0 \times 10^{-7} \\
-7.0 \times 10^{-7}\end{array}$ & $\begin{array}{l}+1.6 \times 10^{-5} \\
+1.5 \times 10^{-5} \\
+8.0 \times 10^{-7} \\
+8.0 \times 10^{-7} \\
\end{array}$ & $\begin{array}{l}+2.5 \times 10^{-2} \\
+2.7 \times 10^{-2} \\
+1.8 \times 10^{-3} \\
+1.9 \times 10^{-3} \\
\end{array}$ \\
\hline E & .05 & $\begin{array}{l}+1.6 \times 10^{-5} \\
+1.8 \times 10^{-5} \\
+1.0 \times 10^{-6} \\
+1.1 \times 10^{-6} \\
\end{array}$ & $\begin{array}{c}+6.0 \times 10^{-9} \\
+5.0 \times 10^{-9} \\
0^{(1)} \\
0^{(1)}\end{array}$ & $\begin{array}{l}-2.5 \times 10^{-8} \\
-2.3 \times 10^{-8} \\
-1.0 \times 10^{-9} \\
-1.0 \times 10^{-9}\end{array}$ & $\begin{array}{l}-6.3 \times 10^{-5} \\
-6.9 \times 10^{-5} \\
-4.1 \times 10^{-6} \\
-4.3 \times 10^{-6}\end{array}$ \\
\hline$y\left(x_{n}\right)$ & & 54.5981500 & .0183156 & .0183156 & 54.5981500 \\
\hline
\end{tabular}

Remarks. (1) The errors for $\operatorname{II}(a=1)$ are diverging from the exact solution for methods A and B (simple von Neumann polynomial), although not as markedly for $h=0.05$.

(2) Methods $\mathrm{A}$ and $\mathrm{C}$ are order 3 while $\mathrm{B}$ and $\mathrm{D}$ are order 4.

(3) Method $\mathrm{E}$ requires $r^{2}$ as many kernel evaluations as the others for a given stepsize, $h$. 
The results in this paper are a summary of the author's Ph.D. thesis written at Dalhousie University. Families of quadrature rule methods for the numerical solution of Volterra integral equations of the first kind have been constructed.

The question of existence, for arbitrary $r$, hinges on the possibility of whether one can construct polynomials $\rho(z)$ (which are Schur or simple von Neumann polynomials) by choosing $p+2-r$ (the number of free parameters) sufficiently large.

Department of Communications

Box 11490-Station $\mathrm{H}$

Ottawa, Ontario K2H 8S2, Canada

1. H. BRUNNER, "The solution of Volterra integral equations of the first kind by piecewise polynomials," J. Inst. Math. Appl., v. 12, 1973, pp. 295-302.

2. W. A. COPPEL, Stability and Asymptotic Behaviour of Differential Equations, Heath Mathematical Monographs, Boston, Mass., 1965. $196-213$

3. R. J. DUFFIN, “Algorithms for classical stability problems," SIAM Rev.,v. 11, 1969, pp.

4. R. A. FUCHS \& V. I. LEVIN, Functions of a Complex Variable and Some of Their Applications, Vol. II, Pergamon Press, Oxford, 1961.

5. C. J. GLADWIN \& R. JELTSCH, "Stability of quadrature rules for first kind Volterra integral equations," $B I T$, v. 14, 1974, pp. 144-151.

6. C. J. GLADWIN, Numerical Solution of Volterra Integral Equations of the First Kind, Ph.D. thesis, Dalhousie University, 1975.

7. P. HENRICI, Discrete Variable Methods in Ordinary Differential Equations, Wiley, New York, 1962.

8. E. ISAACSON \& H. B. KELLER, Analysis of Numerical Methods, Wiley, New York, 1962.

9. M. KOBAYASI, "On the numerical solution of the Volterra integral equation of the first kind by the trapezoidal rule," Rep. Statist. Appl. Res. Un. Japan. Sci. Engrs., v. 14, 1967, pp. 1-14.

10. J. J. H. MILLER, "On the location of zeroes of certain classes of polynomials with applications to numerical analysis," J. Inst. Math. Appl., v. 8, 1971, pp. 397-406.

11. W. POGORZELSKI, Integral Equations and Their Applications, Vol. 1, Pergamon Press, Oxford, 1966.

12. A. RALSTON, A First Course in Numerical Analysis, McGraw-Hill, New York, 1965.

13. R. WEISS, Numerical Procedures for Volterra Integral Equations, Ph.D. thesis, The Australian National University, Canberra, 1972. 